Ibn Al-Haitham Jour. for Pure \& Appl. Sci. 33 (4) 2020

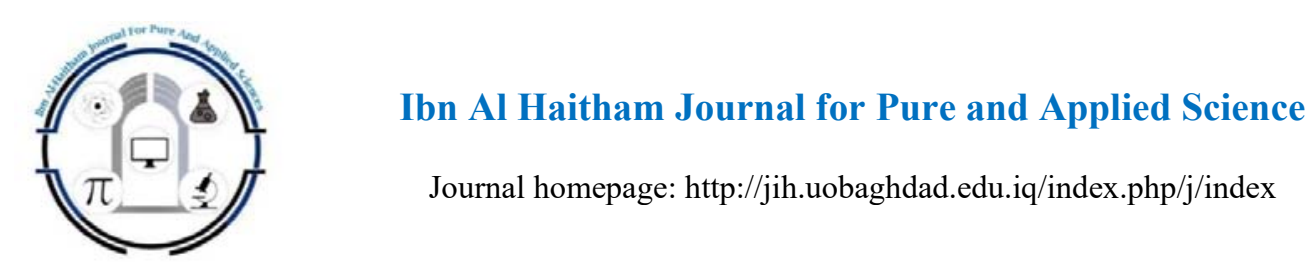

\title{
Performance Enhancement of Face Recognition under High-Density Noise Using PCA and De-Noising Technique
}

\author{
Nada Jasim Habeeb \\ Middle Technical University \\ nadaj2013@gmail.com
}

Article history: Received 19 May 2020, Accepted 28 June, Published October 2020.

Doi: $10.30526 / 33.4 .2527$

\begin{abstract}
Abstarct
There are many techniques for face recognition which compare the desired face image with a set of faces images stored in a database. Most of these techniques fail if faces images are exposed to high-density noise. Therefore, it is necessary to find a robust method to recognize the corrupted face image with a high density noise. In this work, face recognition algorithm was suggested by using the combination of de-noising filter and PCA. Many studies have shown that PCA has ability to solve the problem of noisy images and dimensionality reduction. However, in cases where faces images are exposed to high noise, the work of PCA in removing noise is useless, therefore adding a strong filter will help to improve the performance of recognizing faces in the case of existing high-density noise in faces images. In this paper, Median filter, Hybrid Median Filter, Adaptive Median filter, and Adaptive Weighted Mean Filter were used to remove the noise from the faces images, and they were compared in order to use the best of these filters as a pre-processing step before the face recognition process. Experimental results showed that the Adaptive Weighted Mean Filter gave better results compared with the other filters. Thus, the performance of face recognition process was improved under high-density noise using the Adaptive Weighted Mean Filter and Principal Component Analysis. For the corrupted images by $90 \%$ noise density, Recognition rate by using Median Filter reached $0 \%$ and $33 \%$ by using Hybrid Median Filter. While Recognition rate by using the Adaptive Median Filter and Adaptive Weighted Mean Filter reached $100 \%$.
\end{abstract}

Keywords: Hybrid Median Filter, Adaptive Median Filter, Adaptive Weighted Mean Filter, PCA, Face Recognition, High-Density Noise. 


\section{Ibn Al-Haitham Jour. for Pure \& Appl. Sci. 33 (4) 2020}

\section{Introduction}

Face recognition has become the basis in many fields due to the flexibility and high degree of security in recognition of the real personality because it relies on personality traits in human face recognition. Face recognition is one of the methods of biometric that has high discrimination accuracy and it is applied in applications such as field confidentiality, image processing, computer vision, access control, video surveillance, etc. The most recent developments in face recognition can be found in [1]. However, sometimes the noise occurs in the face image due to various sources, including noise caused by a malfunction in the imaging equipment used, noise due to an error in the process of data transfer which leads to the emergence of spots or points sporadic randomness in the image and is dark or light, and called the salt and pepper, or noise resulting from image acquisition which is called Gaussian noise $[2,3]$. In the case of severely deformed in the face image because the image is exposed to high intensity noise, it is difficult to recognize the face. The following section presents literature review of the most recent face recognition techniques using de-noising filter and PCA.

The paper is organized as follows: Section 2 reviews the previous literature related to this work. In Sections 3 and 4, the theoretical topics used in this work are presented. The suggested face recognition method based on de-noising filter and PCA is described in Section 5 describes. In section 6, the experimental results are presented. Finally, Section 7 presents the conclusion.

\section{Related works}

In [4], the author presented a face recognition method based on Binary Rotation Invariant and Noise Tolerant Euclidean (BRINE) descriptor. Principal Component Analysis (PCA) is used for reduction of time complexity in image on BRINE features. Support vector machine is used to predict the input face.

Faces images that contain noise that adversely affect the accuracy of face recognition are a major challenge. The authors in [5] suggested a face recognition method using a deep neural network to distinguish faces under noise. They introduced a multi-input structure in the final layer of the proposed network to extract a multi-scaled feature and more distinct from the input image. In [6], the authors proposed a face recognition technique for noisy images based on Pulse Coupled Neural Networks, Principal Component Analysis, and Support Vector Machine. For clustering the characteristic area of noisy face image, Pulse Coupled Neural Network was used. Then Principal Component Analysis was used for feature extraction. Finally, Support Vector Machine was applied for human face classification and recognition. The author in [7] suggested a method to reduce the noise of the image before the face recognition process. PCA was applied to extract two sub-images, one with intensity variance with fewer features and the other with higher intensity variance congaing big features. The kernel PCA was applied on the first sub-image to reproduce the image details and a denoising filter was applied to the second sub-image to get a noise-free image. The resulted image was used in the recognition process for better performance. The authors in [8] proposed a face recognition system by integrating neural network, automatic decoding and noise reduction. Automatic encryption was used to create a neural network that learns the identification function by setting restrictions to learn accurate input representations. 


\section{Ibn Al-Haitham Jour. for Pure \& Appl. Sci. 33 (4) 2020}

Despite the good results of these face recognition systems as they have adapted under specific conditions in the low-density of noise, they fail and do not produce the same results in different circumstances. Therefore, the efficiency of the face recognition system depends on its ability to recognize faces under changes in the face image due to exposure to high-density noise. In order to perform more robust face recognition under high density noise, the appropriate de-noising filter is applied as a preprocessing step before face recognition process. This will lead to the enhancement of the performance of the face recognition. The steps of the face recognition process based on de-noising technique and PCA can be defined as follows: (1) Read the corrupted face image (test image). (2) Image de-noising filter is applied to the corrupted image. (3) Face recognition is performed using PCA as a feature extraction. (4) Compute the distance between the feature vector of the de-noised test image and feature vectors of the training images. (5) Finally, retrieve the face image from the training database that has a minimum distance to test image.

\section{Image De-noising Filter}

Digital image is a matrix containing grey-level or color pixel intensities. The noise model can be defined as:

$f(q)=I(q)+n(q)$,

where $I(q)$ is the clean pixel value and $n(q)$ is the noisy pixel value at location $q$. In order to evaluate the performance of the proposed method, this section provides a brief view of the most popular noise removal techniques which are Median Filter, Hybrid Median Filter, Adaptive Median Filter, and Adaptive Weighted Mean Filter.

\subsection{Median Filter}

Median Filter (MF) is the basic and simplest filter to remove noise from a noisy image. Noisy pixel is replaced by the median value of an arranged sequence of neighboring pixels. The number of neighbors is determined by the size of the window [9]. The MF can be expressed mathematically as in the following equations:

$M D(q)=$ Median $\{q i\}$

$M D(q)=q i(N+1) / 2$,

when $N$ is odd

$M D(q)=1 / 2[q i(N / 2)+q i(N / 2)]$,

when $N$ is even

$q 1, q 2, q 3, \ldots, q N$ is the sorted sequence of neighbor pixels

\subsection{Hybrid Median Filter}

Hybrid Median Filter (HMF) removes the noise with preserving edges. Compared with the MF, the HMF has a better property to maintain corner characteristics. The HMF can be defined as:

$\operatorname{HMF}(q)=\left\{\begin{array}{c}\operatorname{Median}\{f(q), q \in N-4(q)\}, \\ \operatorname{Median}\{f(q), q \in \operatorname{Cross} N-4(q)\}, \\ f(q) .\end{array}\right.$ 


\section{Ibn Al-Haitham Jour. for Pure \& Appl. Sci. 33 (4) 2020}

In HMF, $q$ is the point of the pixel value which can be replaced with: (1) the median of the median of Four-Neighborhood of the point $q$, (2) the median of cross neighbors of the point $q$, (3) the pixel value of the point $q[10]$.

\subsection{Adaptive Median Filter}

Adaptive Median Filter (AMF) is widely used in many applications. The work of this filter is similar to the work of MF filter, but it has a number of several features:

(1) Pixels are separated into clean and noisy pixels. (2) It uses a dynamic size window whose size changes according to the amount of noise. (3) This filter works well for the images subjected to low-intensity noise as well as for the images subjected high-intensity noise [11].

\subsection{Adaptive Weighted Mean Filter}

Adaptive Weighted Mean Filter (AWMF) removes high-intensity noise from noisy images. This filter expands the window size until the minimum and maximum values for two consecutive windows are equal. The pixel is considered noisy pixel if it is equal to the maximum or minimum values, otherwise it is considered clean pixel. Noisy pixel is replaced by the weighted mean of the current window. The clean pixels remain unchanged [12].

\section{Face Recognition based on Principal Component Analysis}

Feature extraction process is a very important step, as it extracts data from an image that is close to the original image [13]. This will facilitate the process of discovering interesting patterns. There are two methods of extracting features: the first method is called holistic feature extraction in which the feature is extracted from the global image and will be as an entry to the recognizing or matching step. The second method is called a local feature extraction, the feature like eye, nose, mouth, etc. These local features will be as an entry to the recognizing or matching step. Principal Component Analysis (PCA) is one of the methods to extract the holistic feature from the image [14-19]. The PCA algorithm is used in the extraction of features in many applications such as pattern recognition, machine learning, image retrieval, face recognition and others [20]. A comprehensive review of PCA can be found in $[21,22]$. The PCA algorithm is used for face recognition by extracting the principal components to express the image of human face and to reduce the dimension of the face image to obtain a low dimension of the space. In general, PCA algorithm extracts the global and low dimensional features [23]. The algorithm of the face recognition using PCA can be found in [24- 26].

\section{De-Noising Filter and PCA Based Face Recognition}

In order to enhance human face recognition under high density-noise, the de-noising filter can be used to remove the noise from the corrupted images and then the feature extraction is performed by using PCA. The PCA is used in this work because it is used for dimensionality reduction and it is one of the possible solutions to solve the problem of noisy data $[27,28]$. The operations of the suggested face recognition process can be illustrated briefly as follows:

1. Load M training images. Each image has a size of $N \times N$ pixels

2. Convert each $2 \mathrm{D}$ image into $1 \mathrm{D}$ vector $\Gamma_{1}, \Gamma_{2}, \ldots, \Gamma_{M}$

3. Compute the average of image vectors by $\psi=\frac{1}{M} \sum_{n=1}^{M} \Gamma_{n}$

4. Find the difference between each image and the average image by $\Phi_{i}=\Gamma_{i}-\psi$

5. Compute the Covariance Matrix (C) to find the feature vectors by 
Ibn Al-Haitham Jour. for Pure \& Appl. Sci. 33 (4) 2020

$C=\frac{1}{M} \sum_{n=1}^{M} \Phi_{i} \Phi_{n}^{T}=A A^{T}$, where $\mathrm{A}=\left\{\Phi_{1}, \Phi_{2}, \ldots, \Phi_{M}\right\}, \mathrm{C}$ is a matrix with size $N^{2} \times N^{2}$ pixels.

6. From $\mathrm{C}$, find $M^{\prime}$ eigenvectors of size $\left(N^{2}\right)$ pixels with the highest eigenvalues, which are called feature vectors or feature space of the training images. $W=[\mathrm{w} 1 \ldots \mathrm{w} 2]$ is assumed as a vector of points in feature space associated with each face image in training set.

7. Read the corrupted image $I_{\text {test }}$ by noise.

8. Apply the de-noising filter on the noisy $I_{\text {test }}$ to remove noise from the image. The result is $I_{\text {clean }}$ image.

9. From the de-noised image $\left(I_{\text {clean }}\right)$, obtain feature space or feature vectors by computing the point $W_{\text {test }}$ using the above formulas.

10. Compute the distance between feature vector of the test image and feature vectors of the training images. Dis $=\min \left\|W_{\text {test }}-W_{k}\right\|$, where $W_{k}$ is a point in feature space of training images. Euclidean distance can be used to find the distance.

11. Show the face image from the training dataset that has a minimum distance with the test image.

Figure 1 illustrates the flowchart of the face recognition method based on de-noising filter and PCA.

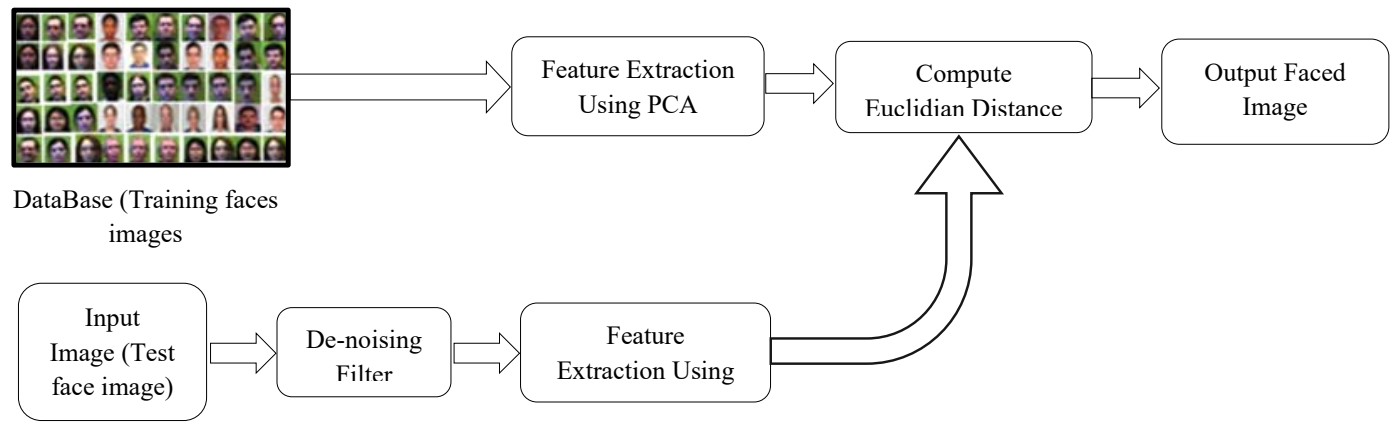

Figure1. Block diagram of the face Recognition based on De-noising Filter and PCA.

\section{Experiment Results}

The performance of the combination of de-noising filter and PCA algorithm for the face recognition have been tested on a free database (facial image database) which was used for research purposes and presented at the site of Essex University (https://cswww.essex.ac.uk/mv/allfaces/). Each image was formatted in 24 colored JPEG and camera used was S-VHS camcorder. The figure 2 shows a sample of the faces images which were considered as a training data set in this work. This database contains faces images of male and female subjects in separate directories with the size of $180 \times 200$ pixels for each image. 
Ibn Al-Haitham Jour. for Pure \& Appl. Sci. 33 (4) 2020

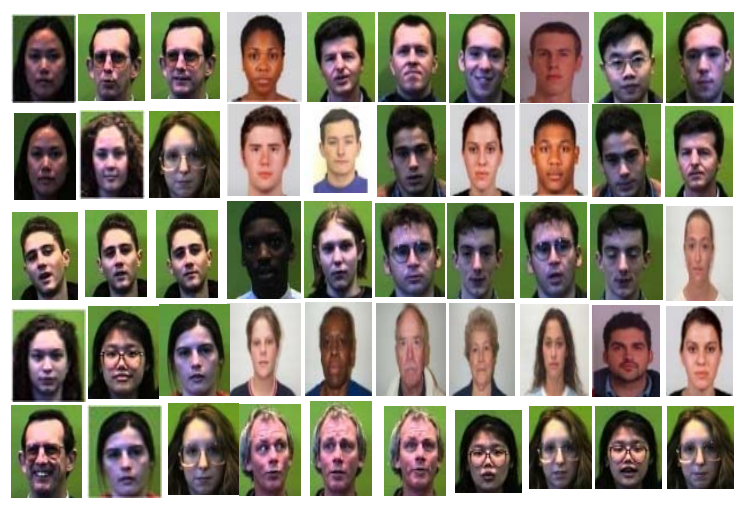

Figure 2. A sample of faces images (training dataset) from faces94 databases

To evaluate the behaviour of the face recognition algorithm, the experiments were achieved on the training (50 images) and testing data sets (6 images). Each original image in the testing data set was corrupted by salt and pepper noise with different noise intensities $(10 \% ; 50 \% ; 90 \%)$. The figure 3 shows the visual results of the corrupted images by different noise intensities.

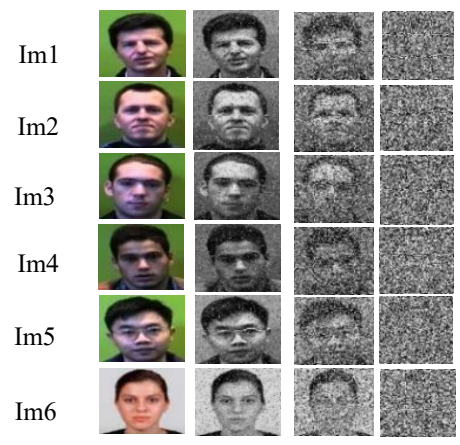

(a) (b) (c) (d)

Figure 3. (a) the original images, (b) the corrupted images with noise intensity $10 \%$, (c) the corrupted images with noise intensity $50 \%$, and (d) the corrupted images with noise intensity $90 \%$.

From Figure 3, adding $90 \%$ density of the salt and pepper noise to the image causes severe damage to the image. The corrupted images can be cleaned by using different de-noising techniques. Figure 4 shows the visual comparison of de-noising techniques performed on the test images.

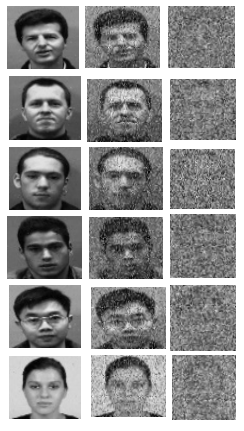

(a)

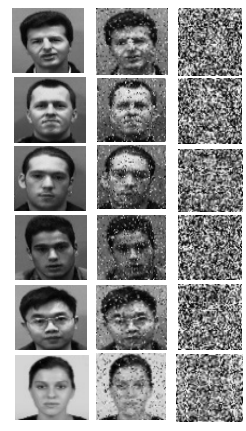

(b)

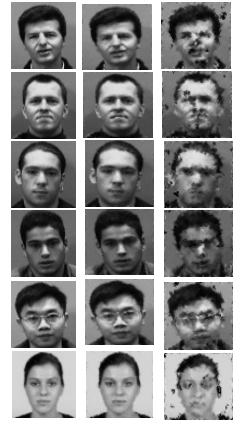

(c)

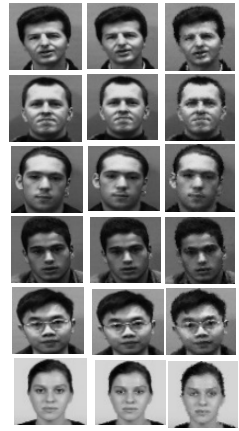

(d)

Figure 4. the comparison results of de-noising the corrupted faces images using (a) MF, (b) HMF, (c) AMF, and (d) AWMF 


\section{Ibn Al-Haitham Jour. for Pure \& Appl. Sci. 33 (4) 2020}

From the above figure, it can be observed that the AWMF filter gives the better results compared with the other filters. Peak Signal to Noise Ratio (PSNR) is used as metric to measure the image quality. To obtain a better face recognition algorithm, the value of PSNR for gray scale image should be maximum. Higher the PSNR, higher quality in the de-noised images.

$$
\begin{gathered}
\text { PSNR }=20 \log _{10}\left(\frac{\operatorname{Max}_{f}}{\sqrt{M S E}}\right) \\
M S E=\frac{1}{v u} \sum_{i=0}^{v-1} \sum_{j=0}^{u-1}\|f(i, j)-g(i, j)\|^{2}
\end{gathered}
$$

Where Mean Squared Error is represented as MSE, $f$ is the two dimension data of the original image, $g$ is the two dimension data of the degraded image in question $v$ and $u$ are the numbers of rows and columns of pixels of the images respectively. $i$ and $j$ represent the indexes of the row and column respectively. $\operatorname{Max}_{f}$ is the maximum value in the original image (clean image). Table 1 to Table 4 illustrate the comparison of PSNR values for the restored images, which were affected by different noise densities, using MF, HMF, AMF, and AWMF filters respectively.

Table 1. the comparison values of PSNR for the restored images using MF

\begin{tabular}{llll}
\hline \multicolumn{1}{c}{ Noise } & $\mathbf{1 0 \%}$ & $\mathbf{5 0 \%}$ & $\mathbf{9 0 \%}$ \\
Image & & & \\
\hline Im1 & 17.6938 & 12.0136 & 9.9451 \\
Im2 & 18.0800 & 12.1232 & 9.9506 \\
Im3 & 18.1177 & 12.1382 & 9.9413 \\
Im4 & 17.9518 & 12.0134 & 9.9351 \\
Im5 & 17.4907 & 11.9717 & 9.9570 \\
Im6 & 16.4798 & 11.5605 & 9.9027 \\
\hline
\end{tabular}

Table 2. the comparison values of PSNR for the restored images using HMF

\begin{tabular}{llll}
\hline \multicolumn{1}{c}{ Noise } & $\mathbf{1 0 \%}$ & $\mathbf{5 0 \%}$ & $\mathbf{9 0 \%}$ \\
Image & & & \\
\hline Im1 & 34.6109 & 14.3554 & 6.2502 \\
Im2 & 35.9293 & 14.2834 & 6.2186 \\
Im3 & 35.7711 & 14.7425 & 6.1834 \\
Im4 & 36.2320 & 14.5863 & 6.1669 \\
Im5 & 34.5701 & 14.4894 & 6.1820 \\
Im6 & 33.7447 & 13.9790 & 5.8894 \\
\hline
\end{tabular}

\begin{tabular}{|c|c|c|c|}
\hline Image & $10 \%$ & $50 \%$ & $90 \%$ \\
\hline Im1 & 40.4054 & 28.1009 & 17.4857 \\
\hline $\operatorname{Im} 2$ & 39.7362 & 27.6846 & 17.5126 \\
\hline $\operatorname{Im} 3$ & 39.8683 & 28.3658 & 18.3798 \\
\hline Im4 & 41.7850 & 29.6597 & 20.3185 \\
\hline $\operatorname{Im5}$ & 37.2654 & 26.9900 & 17.9901 \\
\hline Im6 & 39.7747 & 27.0106 & 15.8127 \\
\hline
\end{tabular}

Table 3. the comparison values ofPSNR for the restored images using AMF 
Ibn Al-Haitham Jour. for Pure \& Appl. Sci. 33 (4) 2020

Table 4. the comparison values of PSNR for the restored images using AWMF

\begin{tabular}{|c|c|c|c|}
\hline Image & $10 \%$ & $50 \%$ & $90 \%$ \\
\hline Im1 & 40.0252 & 34.9009 & 25.6418 \\
\hline $\operatorname{Im} 2$ & 41.3485 & 35.8345 & 26.2244 \\
\hline $\operatorname{Im} 3$ & 41.8706 & 36.6345 & 27.4689 \\
\hline Im4 & 42.6152 & 37.5417 & 28.2581 \\
\hline $\operatorname{Im5}$ & 39.3059 & 33.8882 & 25.1361 \\
\hline Im6 & 39.5629 & 34.3306 & 24.8526 \\
\hline
\end{tabular}

From the tables above, the PSNR values for the restored images using AWMF have better values compared with the other values of PSNR. The evaluation of performances of different methods (MF-PCA, HMF-PCA, AMF-PCA, and AWMF-PCA) is presented using the recognition rate for the comparison. Table 5 illustrates the experimental results on recognition rates for the different face recognition methods based on de-noising filters and PCA.

Table 5.the comparison of the recognition rate of the face recognition based on de-noising filter and PCA

\begin{tabular}{llll}
\hline \multicolumn{1}{c}{ Recognition Rate } & $\begin{array}{l}\text { De-noising } \\
\text { Corrupted } \\
\text { images with } \\
\text { noise 10\% }\end{array}$ & $\begin{array}{l}\text { De-noising } \\
\text { Corrupted } \\
\text { images with } \\
\text { noise 50\% }\end{array}$ & $\begin{array}{l}\text { De-noising } \\
\text { Corrupted } \\
\text { images with } \\
\text { noise 90\% }\end{array}$ \\
\hline MF-PCA & $\% 100$ & $\% 83$ & $\% 0$ \\
HMF-PCA & $\% 100$ & $\% 100$ & $\% 33$ \\
AMF-PCA & $\% 100$ & $\% 100$ & $\% 100$ \\
AWMF-PCA & $\% 100$ & $\% 100$ & $\% 100$ \\
\hline
\end{tabular}

From Table 5, we can see the face recognition based on AWMF-PCA and AMF-PCA has greater values in the recognition rate compared with other methods. Using one of these denoising filters, AWMF and AMF, as preprocessing step in face recognition algorithm will improve the performance of the face recognition under high-density noise. The face recognition based on AWMF-PCA and AWMF-PCA gives recognition rate values equal $100 \%$ under low and high noise densities.

\section{Conclusion}

In this paper, the problem of face recognition for the face's images corrupted with highdensity noise has been addressed. The popular noise removal filters (Median filter, Hybrid Median Filter, Adaptive Median filter, and Adaptive Weighted Mean Filter) were used. A comparison was made for the purpose of finding the best filter to be used as a preprocessing step before the face recognition process using the PCA and matching process. Numerous studies have demonstrated the ability of the PCA to reduce noise from images in addition to reduce dimensional space. Results of comparison between filters showed that the best performance of the de-noising filter was the Adaptive Weighted Mean and then the Adaptive Median filter came after it compared with the other two filters. Therefore, it is possible to use one of these two filters as a preprocessing step before recognizing faces. This will help to 


\section{Ibn Al-Haitham Jour. for Pure \& Appl. Sci. 33 (4) 2020}

improve the face recognition performance in the event that faces images which are exposed to high noise. In the future work, the problem of noisy face image with high density noise can be solved by using Deep Learning. This will greatly enhance and improve the performance of face recognition under high-density noise.

\section{Acknowledgements}

In this work, the database of face images, which have been provided by Essex University, were used in order to analyze the behavior of the performance of the face recognition under high density noise. Thanks to Essex University for supporting the researcher.

\section{References}

1. 1.Jayaraman, U.; Gupta, P.; Gupta, S.; Arora, G.; Tiwari, K. Recent Development in Face Recognition. " Neurocomputing, 2020, doi.org/10.1016/j.neucom.2019,08,110.

2. 2.Deng, X.; Ma, Y.; Dong, M. A new adaptive filtering method for removing salt and pepper noise based on multilayered PCNN. Pattern Recognition Letters, 2016, 79, 817, doi: 10.1016/j.patrec.2016,04,019.

3. 3.Vyas, Aparna, Soohwan Yu, and Joonki Paik, .Multiscale transforms with application to image processing. Springer Singapore, 2018, doi.org/10.1007/978-98110-7272-7.

4. 4.Sreeraj, M. 2015. Texture based multi-view face recognition in noisy images using BRINE feature. International Conference on Computing and Network Communications (CoCoNet). IEEE, 2015.

5. 5.Ding, Y.; Cheng, Y.; Cheng, X.; Li, B.; You, X.; Yuan, X. Noise-resistant network: a deep-learning method for face recognition under noise. EURASIP Journal on Image and Video Processing, 2017, doi:10.1186/s13640-017-0188-z

6. 6.Feifei, S.; Min, H.; Rencan, N.; Zhangyong, W. Noisy faces recognition based on PCNN and PCA. 13th IEEE International Conference on Electronic Measurement \& Instruments (ICEMI). 2017.

7. 7.Berrimi, Fella, Khier Benmahammed, and Riadh Hedli. Denoising of degraded faces images sequence in PCA domain for recognition. Journal of King Saud UniversityComputer and Information Sciences. 2019, doi.org/10,1016/j.jksuci.2019.04,014.

8. 8.Görgel, Pelin.; Ahmet Simsek. 2019. Face recognition via Deep Stacked Denoising Sparse Autoencoders (DSDSA). Applied Mathematics and Computation 355 2019: 325-342. 9.Görgel, P.; Simsek, A. 2019. Face recognition via Deep Stacked Denoising Sparse Autoencoders (DSDSA). Applied Mathematics and Computation, 355, 325 342, doi:10.1016/j.amc.2019.02.071.

9. 10.George, G.; Oommen, R. M.; Shelly, S.; Philipose, S. S.; Varghese, A. M. A survey on various median filtering techniques for removal of impulse noise from digital image. In 2018 Conference on Emerging Devices and Smart Systems (ICEDSS) (235238). IEEE. 2018.

10. Wenpeng, Zhang, ed. Scientia Magna, 2011. International book series. Infinite Study. 7, 4. 2012. 


\section{Ibn Al-Haitham Jour. for Pure \& Appl. Sci. 33 (4) 2020}

11. 11.Habeeb, Nada Jasim. Comparative analysis of Median filter family for Removing High-Density Noise in Magnetic Resonance Images. Iraqi Journal of Science 2019, 2246-2256, doi: 10.24996/ijs.2019,60,10,19.

12. 12.Zhang, Peixuan,; Fang Li. A new adaptive weighted mean filter for removing saltand-pepper noise. IEEE signal processing letters 21.10 2014, 1280-1283, doi: 10.1109/LSP.2014.2333012.

13. 13.Ding, Shifei, Ding, S.; Zhu, H.; Jia, W.;Su, C. A survey on feature extraction for pattern recognition. Artificial Intelligence Review 37.3 2012, 169-180, doi.org/10.1007/s10462-011-9225-y.

14. Kumar, Santosh, Singh, S. K.; Singh, R; Singh, A. K. Animal Biometrics: Techniques and Applications. Springer, 2018, doi: 10.1007/978-981-10-7956-6.

15. 14.Najab, Abida, et al. Classification of settlements in satellite images using holistic feature extraction. 2010 12th International Conference on Computer Modelling and Simulation. IEEE, 2010.

16. 15.Pathan, Al-Sakib Khan, ed. Crowd Assisted Networking and Computing. CRC Press, 2018.

17. Camastra, Francesco, and Alessandro Vinciarelli. Machine learning for audio, image and video analysis: theory and applications. Springer, 2015, doi:10,1007/978-1-84800007-0.

18. 16.Sun, Zhenan, He, R.; Feng, J.; Shan, S.; Guo, Z. (Eds.). Biometric Recognition: 14th Chinese Conference, CCBR 2019, Zhuzhou, China, October 12-13, 2019, Proceedings. 11818. Springer Nature, 2019.

19. 17.Tan, Tieniu, Li, X.; Chen, X.; Zhou, J.; Yang, J.; Cheng, H. (Eds.). Pattern Recognition: 7th Chinese Conference, CCPR 2016, Chengdu, China, November 5-7, 2016, Proceedings.. 662. Springer, 2016.

20. 18. Mohammed, Nwayyin N., et al. 2018. "Face Recognition Based on PCA with Weighted and Normalized Mahalanobis distance." International Conference on Intelligent Informatics and Biomedical Sciences (ICIIBMS). 3. IEEE. 11. 2018.

21. 19.Jolliffe, Ian T,; Jorge Cadima. Principal component analysis: a review and recent developments. Philosophical Transactions of the Royal Society A: Mathematical, Physical and Engineering Sciences 374.2065, 2016: 20150202., doi.org/10.1098/rsta.2015.0202.

22. Velliangiri, S.; Alagumuthukrishnan, S.. A Review of Dimensionality Reduction Techniques for Efficient Computation. Procedia Computer Science, 2019, 165, 104111, doi: 10.5772/18251, doi.org/10.1016/j.procs.2020,01,079.

23. 20.Revina, I. Michael, WR Sam Emmanuel. A survey on human face expression recognition techniques. Journal of King Saud University-Computer and Information Sciences 2018, doi.org/10.1016/j.jksuci.2018,09,002.

24. 21.Dash, Baba (https://www.mathworks.com/matlabcentral/fileexchange/45750-facerecognition-using-pca), MATLAB Central File Exchange. Retrieved May 1, 2020.

25. 22.Lwin, Hteik Htar, Aung Soe Khaing, and Hla Myo Tun. Automatic door access system using face recognition. International Journal of scientific \& technology research. 2016, 4.6: 210-221,doi: 10.1109/I2CT.2018.8529749. 
Ibn Al-Haitham Jour. for Pure \& Appl. Sci. 33 (4) 2020

26. 23.Wang, Chengliang, Lan, L.; Zhang, Y.; Gu, M. Face recognition based on Principal Component Analysis and support vector machine. 3rd International Workshop on Intelligent Systems and Applications. IEEE, 2011.

27. 24.Babu, Y. Murali Mohan, M. Venkata Subramanyam, MN Giri Prasad. PCA based image denoising. Signal \& Image Processing. 2012, 3.2: 236, doi: 10.5121/sipij.2012.3218.

28. 25.Does, M. D.; Olesen, J. L.; Harkins, K. D.; Serradas-Duarte, T.; Gochberg, D. F.; Jespersen, S. N.; Shemesh, N. Evaluation of principal component analysis image denoising on multi-exponential MRI relaxometry. Magnetic resonance in medicine. 2019, 81,6: 3503-3514, doi: 10.1002/mrm.27658. 